Annuaire suisse de politique de développement

5 | 1985

Annuaire Suisse - Tiers Monde 1985

\title{
II. Politique intérieure / Politique extérieure
}

\section{(2) OpenEdition \\ 12 Journals}

Édition électronique

URL : http://journals.openedition.org/aspd/1194

DOI : $10.4000 /$ aspd. 1194

ISSN : 1663-9669

Éditeur

Institut de hautes études internationales et du développement

\section{Édition imprimée}

Date de publication : 1 janvier 1985

Pagination : 65-69

ISSN : 1660-5934

\section{Référence électronique}

" II. Politique intérieure / Politique extérieure », Annuaire suisse de politique de développement [En ligne],

5| 1985, mis en ligne le 15 février 2013, consulté le 08 septembre 2020. URL : http://

journals.openedition.org/aspd/1194; DOI : https://doi.org/10.4000/aspd.1194 


\section{POLITIQUE INTERIEURE / POLITIQUE EXTERIEURE}

\section{DEUXIEME REVISION DE LA LOI SUR L'ASILE}

Depuis 1979, il existe en Suisse une loi générale sur l'asile, dont une première révision est entrée en vigueur le 1er juin 1984; la procédure simplifiée de celle-ci devait permettre d'accélérer le traitement de nombreuses demandes $d$ 'asile. Diverses initiatives parlementaires réclamèrent une nouvelle révision partielle de la loi sur l'asile, de façon à diminuer encore le caractère attirant de la Suisse en tant que pays d'asile. En juillet 1985, le projet de la deuxième révision de la loi a été soumis en consultation.

L'article de M.-C. Caloz-Tschopp, qui figure dans la deuxième partie du présent ouvrage, traite des changements de la politique suisse d'asile par rapport aux flux des réfugiés en provenance du Tiers Monde.

L'accroissement des demandes d'asile de Tamouls provenant du Sri Lanka est au centre des débats et des comptes rendus dans les média; de même que les problèmes dus à l'augmentation de la xénophobie qui en résultent. Une indiscrétion a rendu public un rapport sur la politique d'asile classé "confidentiel", qui était adressé par le ministère public fédéral aux commandants de police cantonaux; il a provoqué de l'indignation et de violentes réactions. Les partis et les œuvres d'entraide ont condamné l'attitude des auteurs du rapport qui "diffamaient en bloc" (1) les demandeurs d'asile. Ce rapport contient une énumération des pratiques abusives ayant trait à l'asile.

A la fin du mois d'avril 1985, il y avait 13661 demandes d'asile en première instance (Office fédéral de police), dans l'attente d'une décision. De plus, il y avait au DFJP et au Conseil fédéral 8333 recours en suspens. En tout, on recensait 30962 réfugiés reconnus en Suisse. Sur les 7435 demandes d'asile soumises en 1984 (7886 en 1983), 2639 des requérants venaient de Turquie et 1236 du Sri Lanka (2).

Un rapport du DFJP sur la situation des Tamouls au Sri Lanka a suscité une vive controverse. En effet, ce rapport déclarait que les Tamouls ne couraient pas de graves dangers au Sri Lanka et qu'on pourrait dorénavant les renvoyer dans leur pays. Le Conseil fédéral a donc décidé en octobre 1984 qu'il était possible en principe de renvoyer au Sri Lanka les Tamouls qui n'étaient pas des réfugiés reconnus. En décembre de la même année, ces mesures de renvoi ont été supprimées en raison de l'aggravation de la situation au Sri Lanka.

Les débats au parlement

Le Conseil des Etats a pris connaissance lors de sa session de mars d'une motion concernant la révision de la loi sur I'asile (Lüchinger, PR), qui avait déjà 
été approuvée en juin 1984 par le Conseil national. Cette motion porte sur les points suivants: le Conseil fédéral est invité à présenter à l'assemblée fédérale un nouveau projet de révision de la loi sur l'asile, permettant de simplifier la procédure de traitement des demandes d'asile, particulièrement lorsqu'il y a des flots de requérants, et de donner au Conseil fédéral une plus grande marge de manoeuvre pour maîtriser les situations exceptionnelles (3). C'est ainsi que le DFJP a été chargé d'élaborer un projet de révision en vue d'une consultation, pour le 30 septembre 1985 au plus tard.

Dans le cadre d'une discussion approfondie sur l'asile politique, le Conseil fédéral a approuvé en juin, avant le début de la session d'été du Parlement, les grandes lignes de la révision de la loi sur l'asile: mesures de simplification accrues et accélération des procédures d'asile, liées à une politique de refoulement conséquente et à une meilleure coopération avec les cantons.

Le DFJP a été chargé de négocier avec les cantons en vue d'une solution globale pour les anciennes demandes d'asile. Certains cantons avaient recours à des pratiques restrictives en matière d'asile. Le canton de Berne par exemple refusait d'accorder une autorisation de travail aux demandeurs d'asile. Le canton de Fribourg n'acceptait plus de demandes d'asile, ce qui était en contradiction avec la loi sur l'asile. Dans le canton des Grisons on demandait des cautions aux requérants d'asile, afin de couvrir les frais d'expulsion (une pratique sans fondement légal). Prenant position sur l'asile politique, I'UDC (Union démocratique du centre) se déclarait en faveur d'une "cantonalisation" des procédures d'asile. A l'avenir, il incombera aux cantons de prendre une décision en première instance. De plus, il faut accorder l'asile collectif aux requérants qui sont déjà très bien assimilés. Les partis bourgeois ( $P R$ et $\mathrm{PDC}$ ) recommandaient une solution globale; le PS a rejeté cette idée pour des motifs constitutionnels.

\section{Projet de révision}

Le projet de révision soumis en consultation en juillet 1985 prévoit les points suivants:

Simplification des procédures: Les requérants d'asile subissent un seul interrogatoire, au lieu de deux, concernant les motifs de leur fuite. Selon la deuxième révision, l'Office fédéral de police peut renoncer à l'interrogatoire et prendre une décision sur la base de documents uniquement. En revanche, les autorités cantonales devraient interroger les demandeurs d'asile de façon approfondie sur les motifs de leur demande.

Détention en vue du refoulement: Pour les requérants d'asile qui, après une décision négative, s'opposent à l'expulsion, on préconise la détention en vue du refoulement (30 jours au maximum). II s'agirait donc de la légalisation de la pratique actuelle.

Aide pour le retour au pays d'origine: La Confédération accorde aux demandeurs d'asile une aide pour le retour dans leur pays d'origine et contribue à 
leur réinsertion. Ce soutien financier doit faciliter le retour du demandeur d'asile dans son pays ou dans un pays qui lui est proche culturellement.

Droit d'urgence: Le Conseil fédéral réclame dans le projet de révision la compétence du droit d'urgence, qui lui permettrait d'intervenir de manière restrictive dans le cas d'une hausse soudaine et massive du nombre des requérants d'asile.

Devoir d'accueil des cantons: Le projet prévoit une compétence subsidiaire du Conseil fédéral quant à la répartition des requérants dans les cantons, en cas d'échec des négociations intercantonales.

Règlement global des demandes: Les requérants d'asile entrés en Suisse avant 1982 et qui n'ont pas encore reçu de réponse à leur demande devraient obtenir un permis de séjour, selon la procédure habituelle de la police des étrangers. Cette disposition s'appliquerait avant tout aux familles.

\section{Proposition des oeuvres d'entraide}

Les oeuvres d'entraide faisant partie de I'Office central suisse d'aide aux réfugiés ont adressé en mai 1984 une lettre aux parlementaires, leur demandant de s'abstenir d'une révision supplémentaire de la loi sur l'asile. Les mesures proposées dans cette lettre visaient à accélérer la procédure de règlement des demandes d'asile; entretemps, elles ont été en partie incluses dans la deuxième proposition de révision (combinaison des interrogatoires cantonaux et fédéraux; des dispositions d'encouragement et de soutien pour le retour au pays d'origine).

Les oeuvres d'entraide ont déclaré au Conseil fédéral qu'elles s'opposaient au renvoi des Tamouls au Sri Lanka. Dans leur rapport sur la situation au Sri Lanka, les oeuvres d'entraide en sont arrivées à une conclusion opposée à celle du Conseil fédéral: le renvoi des Tamouls leur paraissait injustifiable. Outre ce rapport sur les problèmes particuliers des Tamouls du Sri Lanka, les oeuvres d'entraide ont présenté une proposition d" "asile à durée limitée" (une année), qui permettrait de préparer convenablement le retour au pays d'origine.

La ligue suisse des droits de l'homme a invité à Lausanne en février 1985 de nombreux représentants de Suisse et de l'étranger à un colloque sur la détérioration de la situation des demandeurs d'asile en Europe. Les participants ont décidé de s'engager collectivement en faveur d'une politique d'asile plus humaine (4).

\section{Notes}

1) Critique de l'Oeuvre suisse d'entraide ouvrière, OSEO, NZZ, 6.6.1985.

2) Chiffres du DFJP, Statistiques sur les étrangers et les demandes d'asile, fin 1984 et fin avril 1985.

3) Motion Lüchinger (PR), NZZ, 12.3.1985. 
4) Le compte rendu du colloque a été publié dans "La Forteresse européenne et les réfugiés", Lausanne, Ed. d'en bas, 1985.

\section{Sources}

DFJP, Révision de la loi sur l'asile: Ouverture de la procédure de consultation, Communiqué de presse du 8.7.1985

DFJP, Avant-projet et rapport sur la révision de la loi sur I'asile, Berne, juillet 1985

Office central suisse d'aide aux réfugiés, Communiqué de presse du 28.5.1984

Caritas Suisse/Action de carême/Pain pour le prochain/Helvetas/Swissaid/EPER, Communiqué de presse du 28.9.1984

Die Wochenzeitung, 14.6.1985

NZZ, 12.3., 4.6. et 6.6.1985

TA, 22.5., 4.6. et 6.6.1985

\section{ADHESION DE LA SUISSE A L'ONU}

En 1984, les deux chambres ont mis un terme aux consultations sur l'éventuelle adhésion de la Suisse à l'ONU. Au printemps, le Conseil national s'est prononcé en faveur de cette adhésion et, au cours de la session d'hiver, le Conseil des Etats en a fait de même. La votation aura lieu au printemps 1986.

Le 15 mars 1984, le Conseil national s'est déclaré favorable à l'adhésion de la Suisse à l'ONU par 112 voix contre 78. Cette décision signifie que le Conseil fédéral devra, avant de voir la Suisse au sein de I'ONU, garantir la neutralité suisse par un certain nombre de déclarations (1).

\section{Débats au Conseil des Etats}

La question de la neutralité était aussi au cœur du débat au Conseil des Etats et représentait I'argument principal des opposants à l'adhésion à I'ONU. Pour ces opposants, une participation de la Suisse aux activités de I'ONU signifierait une menace pour la neutralité. A leur avis, il n'est pas possible d'associer la neutralité suisse à cette "organisation extrêmement politisée" (2) qu'est I'ONU. Le conseiller aux Etats Muheim, président de la commission, représentait les partisans de l'adhésion; il a défini la politique extérieure comme étant un des moyens pour le gouvernement de garantir l'intégrité territoriale, l'indépendance politique et l'identité culturelle. Selon lui, la neutralité sert à défendre ces objectifs, mais elle n'est pas définie dans la Constitution comme un but poursuivi par la Confédération; elle est en fait un instrument qui s'adapte aux transformations du contexte environnant. Les partisans de l'adhésion de la Suisse à l'ONU citent en exemple d'autres Etats neutres comme la Suède, la Finlande et l'Autriche qui participent activement au sein de I'ONU.

Un autre argument en faveur de l'entrée à I'ONU a été cité dans le débat; il s'agit de la contribution que la Suisse pourrait apporter sur le plan de la 
formation du droit économique et du droit international. En effet, si elle devenait membre de I'ONU, la Suisse pourrait participer activement à l'élaboration de nouvelles normes juridiques internationales qu'elle-même devrait dès lors obligatoirement adopter.

A titre de membre de I'ONU, la Suisse pourrait s'exprimer sur des questions importantes lors de l'Assemblée générale des Nations-Unies et exercer une influence directe sur les activités de l'ONU, ce qui est impossible tant qu'elle n'a pas un statut d'observateur.

Après deux jours de débats, le Conseil des Etats a approuvé l'adhésion de la Suisse à I'ONU par 24 voix à 16 .

Les plébiscite aura lieu le 16 mars 1986.

\section{Notes}

1) L'ONU, fondée en 1945, compte à présent 159 Etats membres. La Suisse est représentée auprès des Nations-Unies par un ambassadeur permanent (I'ambassadrice Francesca Pometta). La Suisse est membre de plusieurs organisations affiliées à I'ONU.

2) Le conseiller aux Etats Affolter (PR), porte-parole de la minorité opposée à l'adhésion à I'ONU, lors du débat au Conseil des Etats, cité in NZZ, 13.12.1984.

\section{Sources}

Message du Conseil fédéral au sujet de l'adhésion de la Suisse à l'ONU, 21 décembre 1981 Rapport de gestion du Conseil fédéral en 1984, Berne février 1985

$\mathrm{NZZ}, 13$ et 14.12 .1984

TA, 24.10.1984 et 14.12 .1984 